\title{
Voter model on a directed network: Role of bidirectional opinion exchanges
}

\author{
Sung-Guk Han, ${ }^{1}$ Jaegon Um, ${ }^{2}$ and Beom Jun Kim ${ }^{1, *}$ \\ ${ }^{1}$ BK21 Physics Research Division and Department of Physics, \\ Sungkyunkwan University, Suwon 440-746, Korea \\ ${ }^{2}$ School of Physics, Korea Institute for Advanced Study, Seoul 130-722, Korea
}

\begin{abstract}
The voter model with the node update rule is numerically investigated on a directed network. We start from a directed hierarchical tree, and split and rewire each incoming arc at the probability $p$. In order to discriminate the better and worse opinions, we break the $Z_{2}$ symmetry $(\sigma= \pm 1)$ by giving a little more preference to the opinion $\sigma=1$. It is found that as $p$ becomes larger, introducing more complicated pattern of information flow channels, and as the network size $N$ becomes larger, the system eventually evolves to the state in which more voters agree on the better opinion, even though the voter at the top of the hierarchy keeps the worse opinion. We also find that the pure hierarchical tree makes opinion agreement very fast, while the final absorbing state can easily be influenced by voters at the higher ranks. On the other hand, although the ordering occurs much slower, the existence of complicated pattern of bidirectional information flow allows the system to agree on the better opinion.
\end{abstract}

PACS numbers: 87.23.Ge, 89.75.Fb

Recently, social phenomenon of the opinion formation has been studied through the uses of simple prototypical models such as the Axelord's culture dissemination model [1] and the voter model [2 10]. In particular, the voter model on complex networks has been popularly studied [2 7], reflecting the broad research interest in dynamic behaviors of complex networks [11]. In the language of statistical physics, the voter model has the same symmetry as the Ising model if the Ising spin is replaced by the opinion of a voter who can have two different opinions $\sigma= \pm 1$. Likewise, the opinion formation dynamics in the voter model corresponds to the coarsening process in the Ising system, although the former is driven by the interface noise while the latter by the surface tension in $d$-dimensional lattice 8], resulting in interesting differences [7, 8]. The analytic approaches have been successfully applied for the voter model in the regular $d$-dimensional lattice [9] and in the complex networks [2[4]: in $d$-dimensional regular lattice, the density $\rho$ of active bonds connecting opposite opinions decays to zero only for $d \leq 2$, whereas for $d>2$ the system never approaches completely ordered absorbing state [8, 9]. The voter model on fractal structures with $d<2$ also exhibits the complete ordering [10]. The ordering dynamics of the voter model in various complex networks share common features [2 [5, 7]: if started from randomly distributed opinions, the system first approaches a quasi-stationary state and stays there for a life time $\tau$. As time is elapsed further the system approaches the absorbing state characterized by $\rho=0$. Interestingly, the life time $\tau$ of the disordered quasi-stationary state increases with the network size $N$ [2 5, 7], which makes the complete ordering in the thermodynamic limit impossible, in accord with the study for regular lattices in a very high dimensionality [9]. There is now growing consensus that the or-

\footnotetext{
* Corresponding author beomjun@skku.edu
}

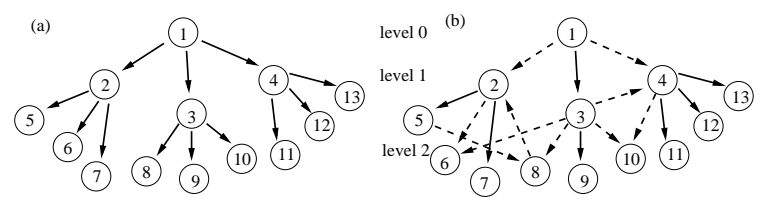

FIG. 1. (a) A directed tree network with the perfect hierarchical structure is initially built with the branching ratio 3 and the number of levels $L=3$. The total number of vertices is given by $N=\left(3^{L}-1\right) / 2$. (b) Each incoming edge is visited one by one, and then split into two edges at the probability $p$ : the existing one but with the half weight and the new one of the half weight from randomly chosen other vertex. Solid (dashed) lines denote the incoming edges of the weight unity (half).

dering dynamics of the voter model depends only weakly on the topological details of the underlying complex networks [3, 7], and that the first and the second moments of the degree distribution mostly determine the ordering behaviors for networks with negligible degree-degree correlation [3, 4]. We emphasize that most existing studies of the voter and other related models have assumed that the networks are undirected. The most recent studies for the ordering dynamics of the voter model have started to consider the directedness of networks [12, 13].

In the sociological and biological disciplines, directed networks are abundant. To name a few, the world wide web, the email communication network, and transcriptional regulatory networks are described better as directed networks. In such directed networks, one generally expects that dynamical behaviors such as synchronization of phase oscillators, opinion formation in the voter model, and the epidemic spread of diseases, exhibit sharp differences from those in undirected networks. In Ref. $\underline{6}$, both the Hodgkin-Huxley model in neuroscience and the sociological voter model have been studied in the small-world type directed network: as more edges 

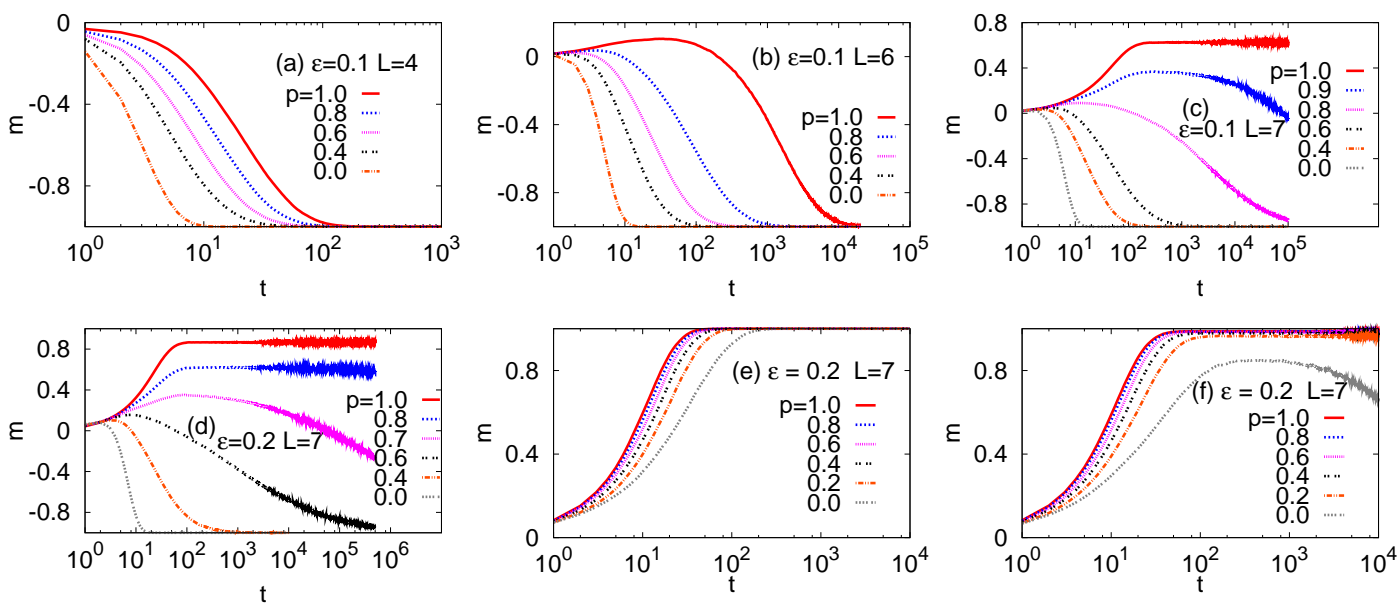

FIG. 2. The order parameter $m(t) \equiv(1 / N)\left\langle\sum_{v} \sigma_{v}\right\rangle$ averaged over 500-2000 independent runs versus the Monte-Carlo time step $t$ for directed network of sizes (a) $L=4(N=40)$, (b) $L=6(N=364)$, (c) $L=7(N=1093)$ with $\epsilon=0.1$ and (d) $L=7$ with $\epsilon=0.2$ at various values of the splitting probability $p$. For small sizes, the voters favor the opinion of the top $\sigma=-1$ at any value of $p$. However, at sufficiently large values of $p$ and for larger sizes, finite fraction of voters begin to have opinions $\sigma=1$ against the top vertex, for a very long time. (e) Undirected network of size $L=7(N=1093)$ with $\epsilon=0.2$ in which all edges are undirected. (f) Different version of undirected network of size $L=7(N=1093)$ with $\epsilon=0.2$ in which all edges are undirected except the ones that connect the top voter and her lower rank direct acquaintances.

become directed, there occur clear changes in dynamic behaviors, and it has been found that bidirectional information exchange helps consensus state to emerge in the voter model. Sánchez et. al. 14] found that the directed links on the directed small-world networks play an important role altering the nature of the phase transition. These findings show that the directedness of networks strongly affects dynamical and critical behaviors of the system.

In this paper we study a sociological game model of voters connected through the directed network structure (see Fig. 11). Our directed network originates from the perfect hierarchical tree structure, which has been found to yield the best synchronizability for the set of identical oscillators [15] but not for the nonidentical oscillators [16]. In order to build directed networks, we start from the tree with $L$ layers and branching ratio $b[L=3$, $b=3$ in Fig. 1(a)]. We then sequentially visit each incoming edge of the weight unity and with the probability $p$ reduce its weight to $1 / 2$ and make a new incoming edge of the weight $1 / 2$ from a randomly chosen vertex. It is to be noted that our way of splitting edges conserves the incoming strength for each vertex and the resulting directed network contains the original tree. When the above procedure is repeated for all incoming edges in the original tree, the resulting network can possess loops [e.g., $2 \rightarrow 5 \rightarrow 8 \rightarrow 2$ in Fig. 1(b)] and directed upward edges [e.g., $8 \rightarrow 2$ in Fig. 1(b)]. Consequently, our network model embeds the hierarchical directed network, which exists abundantly in human societies: Decision makings in corporations, governments, and religious organizations can be described as opinion formation on hierarchical structures [17].
After the construction of directed networks, we perform the voter model simulations on top of the network structures. The original voter model with two opinions $\sigma= \pm 1$ for undirected networks proceeds as follows: A vertex $v$ is chosen at random. One of its neighbors, say $w$, is chosen randomly among $v$ 's direct acquaintances. The opinion $\sigma_{v}$ of the vertex $v$ is changed to that of $w$ s. The $N$ repetitions of the above process constitutes a unit time step so that each voter has chance to update its opinion once on average. The generalization for directed and weighted networks is straightforward: we only need to change the procedure so that $w$ is now chosen only from $v$ 's incoming neighbors $(w \rightarrow v)$ and the weight of this chosen incoming link is used as the probability of acquaintance of $v$ and $w$. Suppose that we play such a voter model on the directed tree structure in Fig. 1(a). The final absorbing state of the whole system is solely determined by the opinion of the top vertex since it does not have incoming edge and thus no one can persuade him otherwise [18]. In other words, if the top vertex has made somehow an incorrect decision, the whole society of voters cannot escape from the disastrous situation in which all voters have the same incorrect opinion of the top vertex. However, in a real experience, one observes that it is plausible that a large scale of consensus of opinions can be made although it is against the opinion of the top. In this regard, we give a distortion in the original voter model as follows: (i) the top voter is assigned the opinion $\sigma_{1}=-1$ which we assume as an incorrect opinion (or poorly decided opinion). All other voters are assigned either 1 or -1 randomly at the initial stage. (ii) A voter $v$ is chosen at random, and one of its incoming neighbor $w$ is also randomly picked. At the probability 
of the weight (either 1 or $1 / 2$ in our model; see Fig. 1) for the link connecting the two, the following procedure is performed. (iii) If $\sigma_{w}(t)=-1$ at time $t, v$ changes its opinion to $w$ 's one, i.e., $\sigma_{v}(t+1)=-1$ at the probability $1-\epsilon$. On the other hand, if $\sigma_{w}(t)=1, v$ always follows $w$ 's opinion, i.e., $\sigma_{v}(t+1)=1$ at the probability unity. In words, the better opinion $\sigma=1$ is always accepted while the slightly worse opinion $\sigma=-1$ is accepted with a bit of hesitation. Within this modified voter model, the original $Z_{2}$ symmetry is broken (compare with Ref. 8) by the asymmetry parameter $\epsilon$ and we drive the system to be in favor of the opinion $\sigma=1$, by giving each voter a slight preference toward $\sigma=1$. The incoming strength, i.e., the sum of the weights of incoming arcs, is conserved in our model, which we interpret as that the total time or resources each voter can use to make contact is fixed to a constant. We have also used the unweighted version of our modified voter model in which the above step (iii) is performed always for the pair $(v, w)$, regardless of the weight of the link. We have observed only insignificant differences and all the results presented in this Brief Report are for the voter model with the edge weight taken into account.

The motivation of our voter model is to mimic realworld situations in which there are two competing options or choices and one is intrinsically better than the other. The worse option can still pervade the whole system, if a dominant agent strongly advocates it. In our model, the intrinsically better option is described by the symmetry breaking parameter $\epsilon>0$, and the strong advocate of the worse option is represented as $\sigma=-1$ assigned for the top voter. The purpose of our Brief report is then to reveal what can be the effects of the connection structure to change the situation so that the better option $\sigma=1$ manages to spread across the whole society.

We construct directed networks of various sizes as described above and perform the simulation of our modified voter model. During the simulation, we measure the order parameter defined as $m(t) \equiv(1 / N)\left\langle\sum_{v} \sigma_{v}(t)\right\rangle$ with $\langle\cdots\rangle$ being the average over network realizations and initial configurations. The consensus state with completely agreed opinion is characterized by either $m=1$ or $=-1$. When the splitting probability $p$ in Fig. 1 is sufficiently small, we expect that the system approaches the consensus state in which all voters have opinion $\sigma=-1$ since the unidirectional information flow is abundant, and very few voters can disobey the opinion of the top voter. In contrast, as $p$ is increased further and thus as lots of directed loops are newly formed, one expects that the situation reverses and a large number of voters will favor the opinion $\sigma=1$. In Fig. 2, the order parameter is displayed as a function of $t$ for various sizes (a) $L=4(N=40)$, (b) $L=6(N=364)$, and (c) $L=7(N=1093)$ at $\epsilon=0.1$, and for comparison (d) $L=7(N=1093)$ at $\epsilon=0.2$ in directed networks, and the undirected cases of (e) and (f) of networks of size $L=7(N=1093)$ at $\epsilon=0.2$ are discussed later. It is clearly seen that the
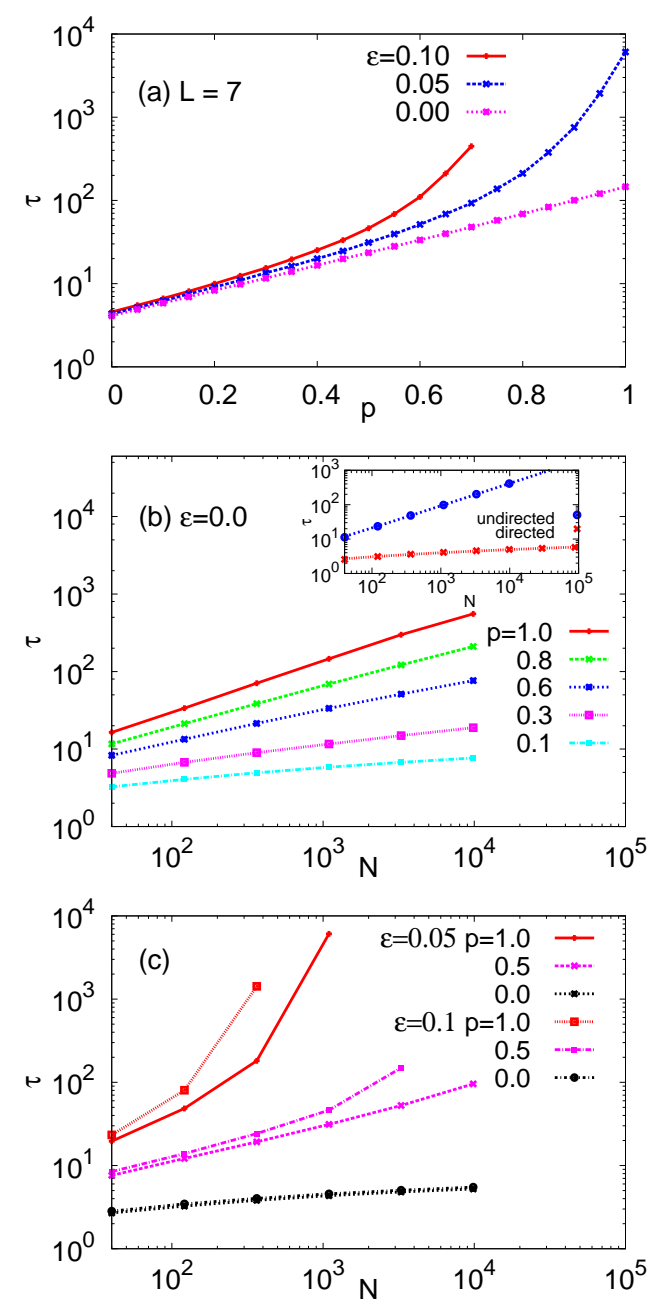

FIG. 3. Relaxation time $\tau$ versus (a) $p$ and [(b) and (c)] $N$. (a) $\tau$ monotonically increases with $p$. (b) For $\epsilon=0.0$, at higher values of $p, \tau$ grows with $N$ algebraically, whereas at lower values of $p \tau$ grows with $p$ logarithmically. The inset exhibits the result of $\tau$ vs. $N$ for directed tree and undirected tree (i.e., where $p=0$ ) and lines are results from the leastsquare-fit to algebraic and logarithmic functions, respectively. (c) For $\epsilon=0.05$ and 0.1 at higher values of $p, \tau$ increases with $N$ faster than algebraic.

above expectation becomes indeed correct as the system size is increased. For small sizes, the system eventually approaches to the absorbing state $m=-1$ at any value of the splitting probability $p$. However, as the size becomes larger [see Fig. 2 (c) and (d) for $L=7(N=1093)$ ] $m$ increases toward positive values at higher values of $p$, indicating that there are more voters with $\sigma=1$ than in the initial state, and $m$ stays at an almost constant value for a very long time [19]. In the later stage, $m$ appears to decay to -1 eventually, very slowly. Furthermore, the larger value of $\epsilon$ makes more voters agree on $\sigma=1$ at lower values of $p$ as shown in Figs. 2 (c) and 2(d). The comparison of the time decay behaviors for different sizes makes us conclude that for even bigger sizes, the system 
will stay at positive values of $m(t)$ in an extremely long time. Due to the limitation of computing resources, it is difficult to decide whether $m(t \rightarrow \infty)=1$ or -1 for large enough value of $p$.

Another interesting observation one can make from Fig. 2 (c) and (d) is that the approach toward $m=-1$ for $p=0$ occurs much faster than toward a positive value of $m$ for $p=1$. This indicates that although the system can achieve the state in which majority of voters keep the better opinion, it takes longer time to get there. We next compute the active bond density $\rho(t)=\left[\sum_{i} \sum_{j \in \mathcal{V}_{i}^{i n}}\left(1-\sigma_{i} \sigma_{j}\right) / 2\right] / \sum_{i}\left|\mathcal{E}_{i}^{i n}\right|$, where $\mathcal{V}_{i}^{i n}$ $\left(\mathcal{E}_{i}^{i n}\right)$ is the set of the incoming nearest vertices (edges) of the $i$ th voter and $\left|\mathcal{E}_{i}^{i n}\right|$ is the number of edges in the set $\mathcal{E}_{i}^{i n}$. The relaxation time to reach the absorbing state $\rho(t)=0$ or $m(t)=-1$ is also calculated. We observe that as $p$ is increased, $\tau$ also increases monotonically, which indicates that the complete opinion agreement to $\sigma=-1$ takes longer time as the network structure becomes more different from the hierarchical structure, and $\tau$ increases more rapidly for the higher value of $\epsilon$, as shown in Fig. 3 (a). For $\epsilon=0.0, \tau$ increases in a logarithmic way with $N$ at $p=0.0$, whereas in undirected tree the relaxation time increases algebraically with $N$ as displayed in the inset of Fig. 3 (b), implying that the consensus of opinions occurs much faster in directed tree networks than in undirected ones. It is notable that the crossover from the logarithmic behavior to the algebraic one exists as $p$ is increased in the case of $\epsilon=0.0$ [see Fig. 3 (b)]. Consequently, we conclude that the bidirectional information flows make the agreement of opinion slower through the formation of a large number of voters against the opinion of the top voter. Figure 3 (c) shows that when $\epsilon>0, \tau$ increases very fast with $N$ for higher values of $p$ (faster than the algebraic increase).
For comparisons, we also study the undirected network version of our model in which the network is constructed in the same way as for directed case (see above) and then all edges are assumed to be symmetric, i.e., bidirectional. We observe that the final consensus state $m(t \rightarrow \infty)>0$ is reached as far as $\epsilon>0$, regardless of the splitting probability $p$, which leads us to conclude that our voter model in directed network and undirected network exhibit a quite different behavior [see Figs. 2 (e) and 2 (f)]. Nevertheless, our main conclusion of the role of the bidirectional information flow is still valid in the undirected networks: the more abundant bidirectional opinion exchanges help the majority of voters to agree on the better opinion.

In summary, we have investigated the simple sociological model of voters on directed networks. Starting from a directed tree structure, each edge weight is halved and a new incoming edge with a half weight is newly connected at the probability $p$. As more edges are split, making the network possess more complicated pattern of information flow channels, it has been found that finite fraction of voters can hold opinions against the opinion of the root voter at the top. This trend becomes more evident as the asymmetry between the two opinions $\sigma= \pm 1$ becomes bigger, making one opinion more favorable than the opposite, and also as $p$ and $N$ become larger. The approach toward the absorbing state has been found to be very slow for large values of $p$. It has also been found that the relaxation time increases very fast with the size $N$.

We acknowledge the support from the Korea Science and Engineering Foundation (Grant No. R01-2007-00020084-0) and the support from Asia Pacific Center for Theoretical Physics (APCTP).
[1] R. Axelrod, J. Conflict Resolut. 41, 203 (1997); C. Castellano, M. Marsili, and A. Vespignani, Phys. Rev. Lett. 85, 3536 (2000); K. Klemm, V. M. Eguíluz, R. Toral, and M. San Miguel, Phys. Rev. E 67, 026120 (2003).

[2] D. Vilone and C. Castellano, Phys. Rev. E 69, 016109 (2004).

[3] F. Vazquez and V. M. Eguíluz, New J. Phys. 10, 063011 (2008).

[4] V. Sood and S. Redner, Phys. Rev. Lett. 94, 178701 (2005).

[5] C. Castellano, D. Vilone, and A. Vespignani, Europhys. Lett. 63, 153 (2003); X. Castelló, R. Toivonen, V. M. Eguíluz, J. Saramäki, K. Kaski, and M. San Miguel, ibid. 79, 66006 (2007); K. Suchecki, V. M. Eguíluz, and M. San Miguel, ibid. 69, 228 (2005); K. Suchecki, V. M. Eguíluz, and M. San Miguel, Phys. Rev. E 72, 036132 (2005).

[6] S. M. Park and B. J. Kim, Phys. Rev. E 74, 026114 (2006).

[7] C. Castellano, V. Loreto, A. Barrat, F. Cecconi, and D. Parisi, Phys. Rev. E 71, 066107 (2005).
[8] I. Dornic, H. Chaté, J. Chave, and H. Hinrichsen, Phys. Rev. Lett. 87, 045701 (2001).

[9] L. Frachebourg and P. L. Krapivsky, Phys. Rev. E 53, R3009 (1996).

[10] K. Suchecki and J. A. Holyst, Physica A 362, 338 (2006).

[11] See, e.g., S. N. Dorogovtsev, A. V. Goltsev, and J. F. F. Mendes, Rev. Mod. Phys. 80, 1275 (2008) and references therein.

[12] N. Masuda and H. Ohtsuki, New J. Phys. 11, 033012 (2009).

[13] M. Á. Serrano, K. Klemm, F. Vazquez, V. M. Eguíluz, and M. San Miguel, J. Stat. Mech.: Theory Exp. (2009), P10024.

[14] A. D. Sánchez, J. M. López, and M. A. Rodríguez, Phys. Rev. Lett. 88, 048701 (2002).

[15] T. Nishikawa and A. E. Motter, Phys. Rev. E 73, 065106(R) (2006).

[16] J. Um, S.-I. Lee, and B. J. Kim, J. Korean Phys. Soc. 53, 491 (2008).

[17] See S. Arbesman, J. M. Kleinberg, and S. H. Strogatz, Phys. Rev. E 79, 016115 (2009), and references therein. 
[18] Similarly, effects of the existence of a single zealot who has more preference for a particular opinion have been studied in M. Mobilia, Phys. Rev. Lett. 91, 028701 (2003)

[19] We also measure the order parameter $m_{S}(t)$ defined as the average over surviving runs, i.e., the time series that decays to the absorbing state $\sigma=-1$ are excluded. As $p$ is increased, $m_{S}(t)$ favors a positive value similarly to $m(t)$. Interestingly, in a broad range of $p, m_{S}(t)$ first decreases and then increases, before it eventually decays to -1 . 